Archive for

Organic Chemistry

Arkivoc 2019, part vi, 386-396

\title{
Synthesis, acaricidal activities and docking study of novel acrylonitrile derivatives
}

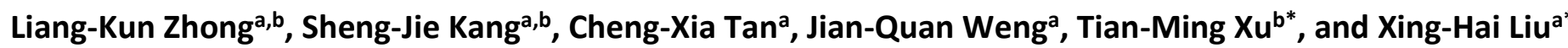 \\ a. College of Chemical Engineering, Zhejiang University of Technology, Hangzhou 310014, China \\ b. Zhejiang Base of National Southern Pesticide Research Centre, Zhejiang Research Institute of Chemical \\ Industry, Hangzhou 310023, China \\ Email:xhliu@zjut.edu.cn; xutianming@sinochem.com
}

Received 09-27-2019

Accepted 12-16-2019

Published on line 01-19-2020

\section{Abstract}

A series of novel acrylonitrile derivatives containing a flexible chain were designed using cyflumetofen as lead compound. They were synthesized via two steps. The structures were confirmed by ${ }^{1} \mathrm{H} N M R,{ }^{13} \mathrm{C} N M R$ and HRMS. The title acrylonitrile derivatives exhibited good acaricidal activity against Tetranchus urticae. In particular, 3j possessed excellent activity in the green house, at the same level as the control Cyflumetofen at 4 days or 7 days.
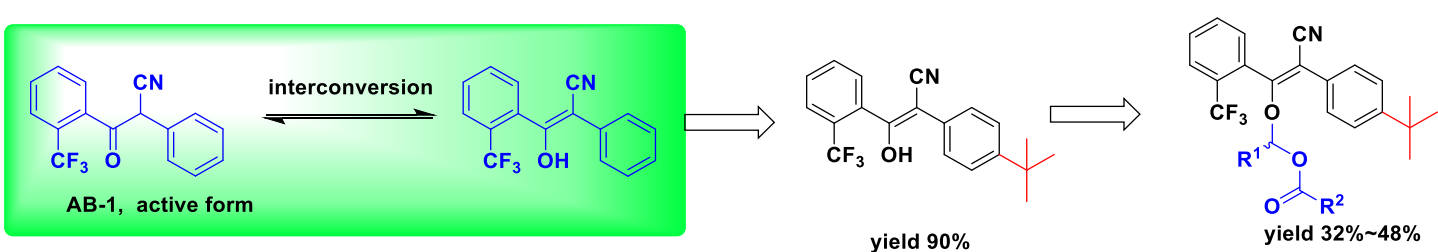

Keywords: acrylonitrile compounds, synthesis, acarcidal activity, SDH 


\section{Introduction}

Plant fungal, weed and insect diseases can decrease productivity of crops in agriculture. ${ }^{1-3}$ Pesticides can control many plant fungal and insect diseases effectively. ${ }^{4-6}$ Mitochondria complex II (Succinate dehydrogenase, SDH) is an important target for pesticidal discovery. ${ }^{5-7}$ To date, many small molecules ${ }^{8-20}$ targeting SDH were discovered as fungicides and insecticides. The acrylonitrile group was a key skeleton in the insecticides, especially in acaricides, such as cyenopyrafen, SYP-9625. Therefore, acrylonitrile compounds are attractive due to novel structures, low toxicity and high activity. Cyflumetofen, a $\beta$-ketonitrile acaricide, that exhibits excellent control effect for spider mites (Oligonychus, Tetranychus and Panonychus), was discovered in 2007 by Otsuka Co., Ltd. Cyflumetofen shows low risk of cross-resistance with other acaricides including bifenazate, propargite and fenpropathrin, and avermectin respectively.

The mechanism (Figure 1) whereby cyflumetofen inhibits respiration of SDH was analysed by pesticidal physiological scientists. ${ }^{21}$ The final metabolite of cyflumetofen is AB-1 (Cyflumetofen de-esterification) and has better activity than cyflumetofen at different concentrations. So the cyflumetofen could be as a pro-pesticide in the acaricide discovery.

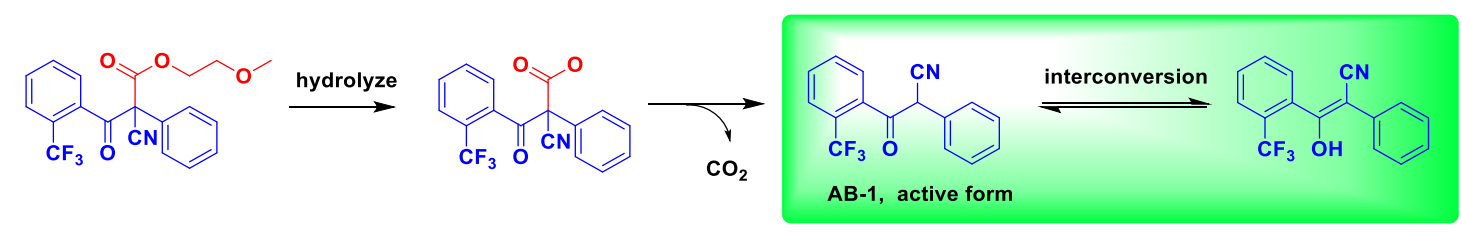

Figure 1. The mode of action of cyflumetofen.

In view of these facts, and also as a part of our work on the synthesis of bioactive lead compounds for drug discovery, ${ }^{22-39}$ the acaricide cyenopyrafen was selected as a lead compound, and a series of cyenopyrafen analogues were designed and synthesized. A flexible chain was introduced onto the $\mathrm{OH}$ group. The acaricidal activity was tested and a docking study was also carried out.

\section{Results and Discussion}

\section{Synthesis and Spectra}

The synthetic route to the title acrylonitrile derivatives is shown in Scheme 1. In the key intermediate 2-(4tert-butylphenyl)-3-hydroxy-3-(2-trifluoromethylphenyl) acrylonitrile 1, the acrylonitrile ( $\mathrm{HO}-\mathrm{C}=\mathrm{C}$ - $\mathrm{CN}$ ) structure can exist either as the enol, or ketone tautomeric forms. From the ${ }^{1} \mathrm{H}$ NMR of 2-(4-tert-butylphenyl)-3hydroxy-3-(2-(rifluoromethylphenyl) acrylonitrile 1, we found two t-Bu signals at $1.30 \mathrm{ppm}$ and $1.36 \mathrm{ppm}$ respectively, with peak areas in the ratio of $1: 1$. The $\mathrm{CHCN}$ signal was found at $5.21 \mathrm{ppm}$. The aromatic signals integrated for 16 , which indicated that the compound 1 contains enol and ketone tautomeric forms. From the ${ }^{1} \mathrm{H}$ NMR data, we can conclude that the ratio of the two tautomeric forms is $1: 1$. For the synthesis of final compounds, there is still a little Z-isomer, which is the same as in related references. ${ }^{40,41}$ The polarity of this Zisomer is small, so it can be easily removed by column chromatography. The residue is the cis product. If we used the potassium salt of intermediate 1 to prepare the final compounds, it still worked well. 


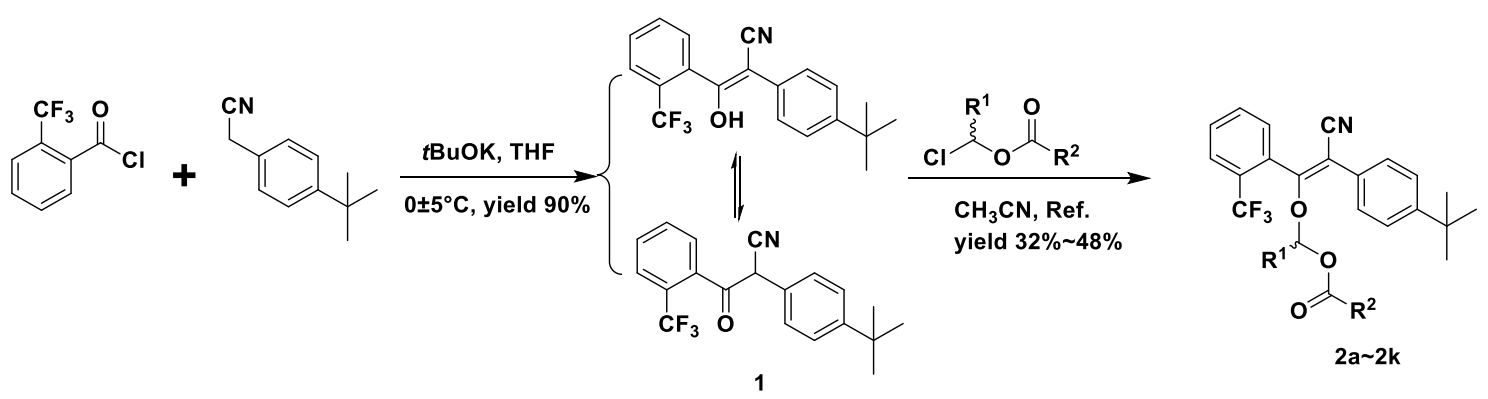

Scheme 1. The synthetic route of title compounds.

All the structures of compounds $\mathbf{2 a - 2} \mathbf{k}$ were determined using ${ }^{1} \mathrm{H}$ and ${ }^{13} \mathrm{C} N M R$, and HRMS analysis. In the ${ }^{1} \mathrm{H}$ NMR spectra, doublets at $\delta 7.4 \mathrm{ppm}$ and $7.7 \mathrm{ppm}$ correspond to the $t$-Bu-ring protons, with a coupling constant of $\mathrm{J} 8.4 \mathrm{~Hz}$. The signals around $7.6 \mathrm{ppm}$ are a multiplet for the protons of the $\mathrm{CF}_{3}$-ring. In the ${ }^{13} \mathrm{C} \mathrm{NMR}$ spectrum, the $\mathrm{CF}_{3}$ showed a quartet signal with a coupling constant of $274 \mathrm{~Hz}$. With regard to the high resolution mass spectra, all the acrylonitrile derivatives showed $\mathrm{M}+\mathrm{H}^{+}$signals with the standard error $m / z \pm$ 0.0030 .

\section{Acaricidal activity}

The acaricidal activity was determined and the results are listed in Table 1. Most of these compounds exhibited excellent activity against Tetranchus urticae, with compounds $\mathbf{2 c}, \mathbf{2} \mathbf{f}$ and $\mathbf{2} \mathbf{g}$ having the best activity (100\%). Compounds $\mathbf{2} \mathbf{a}$ and $\mathbf{2} \mathbf{b}$ also exhibited good activity (>90\%). The compounds $\mathbf{2} \mathbf{d}$ and $\mathbf{2} \mathbf{e}$ had no activity. From these data, we find that when the alkyl group is $t$-Bu or 2-methyl-Bu, the activity is lower. Because of the high acarcidal activity, four compounds were tested for acarcidal activity in the green house. Table 2, shows that these compounds exhibited excellent acarcidal activity at $5 \mathrm{mg} / \mathrm{L}$ or $2.5 \mathrm{mg} / \mathrm{L}$ for 4 days, 7 days or 14 days. The activity is higher than that of control cyflumetofen. At $1.25 \mathrm{mg} / \mathrm{L}$, only compound $\mathbf{2 k}$ exhibited low activity, which is lower than control. Even at $0.625 \mathrm{mg} / \mathrm{L}$, compounds $\mathbf{2} \mathbf{i}$ and $\mathbf{2} \mathbf{j}$ still exhibited moderate activity, which is the same as the control.

Table 1. The acaricidal activities of some acrylonitrile derivatives at $2.5 \mathrm{mg} / \mathrm{L}$

\begin{tabular}{|c|c|c|c|}
\hline No. & $\mathrm{R}^{1}$ & $\mathrm{R}^{2}$ & Mortality(\%) \\
\hline $2 a$ & $\mathrm{CH}_{3}$ & & 93.55 \\
\hline $2 b$ & $\mathrm{H}$ & & 97.47 \\
\hline $2 c$ & $\mathrm{CH}_{3}$ & & 100.00 \\
\hline $2 d$ & $\mathrm{CH}_{3}$ & & 28.07 \\
\hline $2 e$ & $\mathrm{CH}_{3}$ & & 15.38 \\
\hline $2 f$ & $\mathrm{CH}_{3}$ & & 100.00 \\
\hline $2 \mathrm{~g}$ & $\mathrm{CH}_{3}$ & & 100.00 \\
\hline
\end{tabular}


Table 2. The control effect of further synthetic acrylonitrile derivatives at different concentrations

\begin{tabular}{|c|c|c|c|c|c|c|}
\hline \multirow{2}{*}{ No } & \multirow{2}{*}{$\mathrm{R}^{1}$} & \multirow{2}{*}{$\mathrm{R}^{2}$} & \multirow{2}{*}{$\begin{array}{c}\text { Concentration } \\
\mathrm{mg} / \mathrm{L}\end{array}$} & \multicolumn{3}{|c|}{ Control effect(\%) } \\
\hline & & & & $4 d$ & $7 d$ & $14 d$ \\
\hline \multirow{4}{*}{$2 \mathrm{~h}$} & \multirow{4}{*}{$\mathrm{CH}_{3}$} & & 5 & 100 & 100 & 100 \\
\hline & & & 2.5 & 87.69 & 83.88 & 53.99 \\
\hline & & & 1.25 & 69.03 & 63.03 & 0 \\
\hline & & & 0.625 & 33.51 & 35.03 & 0 \\
\hline \multirow{4}{*}{$2 \mathbf{i}$} & \multirow[b]{4}{*}{$\mathrm{CH}_{3}$} & & 5 & 100 & 86.62 & 92.4 \\
\hline & & & 2.5 & 97.86 & 96.25 & 76.12 \\
\hline & & & 1.25 & 77.03 & 74.37 & 2.92 \\
\hline & & & 0.625 & 34.8 & 51.19 & 0 \\
\hline \multirow{4}{*}{$2 \mathrm{j}$} & & & 5 & 100 & 98.42 & 99.92 \\
\hline & \multirow{3}{*}{$\mathrm{CH}_{3}$} & & 2.5 & 100 & 99.26 & 98.77 \\
\hline & & & 1.25 & 96.64 & 96.48 & 54.2 \\
\hline & & & 0.625 & 39.97 & 41.71 & 0 \\
\hline \multirow{4}{*}{$2 k$} & \multirow{4}{*}{$\mathrm{CH}_{3}$} & & 5 & 95.99 & 96.5 & 56.25 \\
\hline & & & 2.5 & 95.13 & 92.54 & 50.92 \\
\hline & & & 1.25 & 34.33 & 49.76 & 47.22 \\
\hline & & & 0.625 & 0 & 64.81 & 0 \\
\hline \multirow{4}{*}{ Cyflumetofen } & & & 5 & 96.74 & 95.45 & 52.58 \\
\hline & & & 2.5 & 76.61 & 71.59 & 53.58 \\
\hline & & & 1.25 & 30.84 & 68.39 & 35.75 \\
\hline & & & 0.625 & 39.9 & 48.02 & 49.04 \\
\hline
\end{tabular}

\section{Molecular docking}

To further explore the interaction of the novel compounds with SDH at the molecular level, a docking study was carried out by using the Discovery studio 2.5. The reported crystal structure of SDH (PDB code: 1ZOY) was selected for docking simulation. As shown in Figure 2, intermediate $\mathbf{1}$ is well-matched to the active pocket of procine SDH. In intermediate 1 there are four hydrogen bonds between carboxyl group, cyano group, trifluoromethyl group, shown by the yellow dotted lines in Figure 2. Firstly, an intermolecular hydrogen bonding is observed between $\mathrm{O}$ of $\mathrm{C}=\mathrm{O}$ and Thr58 with thae distance of $2.3 \AA$. Secondly, a hydrogen bond can be seen between $\mathrm{F}_{\text {of }} \mathrm{CF}_{3}$ group and Gly31 with a length of $2.5 \AA$. Another two intermolecular hydrogen bonds are shown between the nitrogen atom of the cyano group with hydrogen of hydroxyl of Ser230 and amino group of Gly216 with lengths of $2.2 \AA$ and $2.2 \AA$ respectively. 


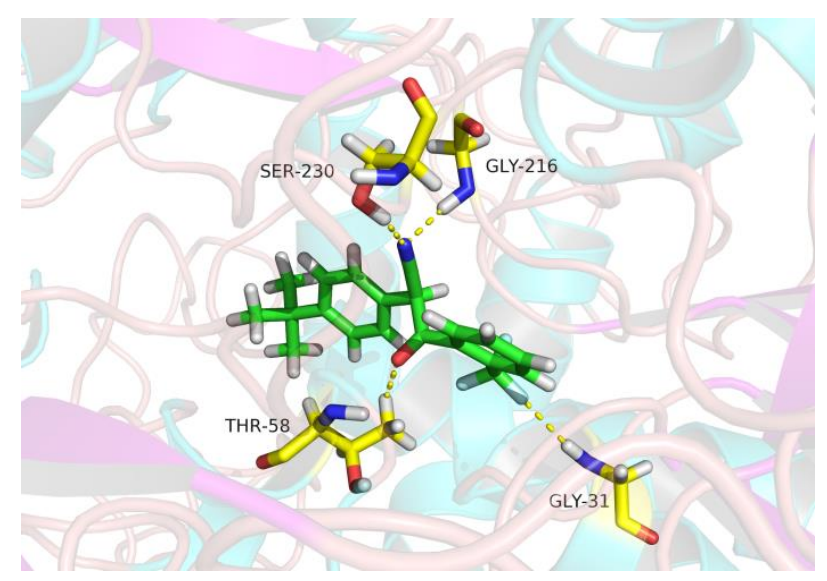

Figure 2. Plot depicting interaction of intermediate $\mathbf{1}$ with active site of SDH.

\section{Conclusions}

A series of novel acrylonitrile derivatives containing a flexible chain were designed, synthesized and characterized. The synthesized compounds were screened for their acaricidal activity against Tetranchus urticae. Among the tested compounds, compound 1-((2-(4-tert-butylphenyl)-2-cyano-1-(2trifluoromethylphenyl)vinyl)oxy)ethyl methyl carbonate $\mathbf{2 j}$ possessed potent acaricidal activity against Tetranchus urticae in the green house in comparison with reference drug cyflumetofen at 4 days or 7 days.

\section{Experimental Section}

General. ${ }^{1} \mathrm{H}$ NMR and ${ }^{13} \mathrm{C}$ NMR spectra were determined on a BRUKER Avance $500 \mathrm{MHz}$ spectrometer using $\mathrm{CDCl}_{3}$ as the solvent. HR-ESI-MS were measured using an Agilent $1100 \mathrm{HPLC}-J E O L$ AccuTOF instrument. All the reagents were analytical grade or synthesized in our lab.

\section{Synthesis}

To a solution of 2-(4-tert-butylphenyl)acetonitrile $(1.73 \mathrm{~g}, 0.01 \mathrm{~mol})$ and $t$-BuOK $(1.232 \mathrm{~g}, 0.011 \mathrm{~mol})$ in THF at $0{ }^{\circ} \mathrm{C}$, 2-trifluoromethylbenzoyl chloride $(2.08 \mathrm{~g}, 0.01 \mathrm{~mol})$ was added dropwise. The mixture was stirred at room temperature. After $2 \mathrm{~h}$, the solvent was evaporated and the residue was treated with $\mathrm{H}_{2} \mathrm{O}(100 \mathrm{~mL})$. The solution was neutralized with $\mathrm{HCl}$ to afford a precipitated solid 1 which, without purification, was filtered off and dried (3.1 g, $90 \%)$.

Synthesis of compound 2a. To a solution of intermediate 1 (3.45 g, $0.01 \mathrm{~mol})$ and $\mathrm{Na}_{2} \mathrm{CO}_{3}(1.59 \mathrm{~g}, 0.015 \mathrm{~mol})$, $\mathrm{Nal}(0.15 \mathrm{~g}, 0.001 \mathrm{~mol})$ in $\mathrm{CH}_{3} \mathrm{CN}(50 \mathrm{~mL})$, the 1-chloroethyl ethyl carbonate $(0.011 \mathrm{~mol})$ was added. The mixture was refluxed for $5 \mathrm{~h}$. After the reaction was completed, the solvent was removed and the residue was purified by column chromatography to give a colourless oil $\mathbf{2 a}(2.1 \mathrm{~g}, 45 \%$-. The following compounds $\mathbf{2 b} \mathbf{b}-\mathbf{2 k}$ were prepared in a similar manner.

1-((2-(4-tert-Butylphenyl)-2-cyano-1-(2-trifluoromethylphenyl)vinyl)oxy)ethyl ethyl carbonate 2a. Yellow oil, yield 45\%, ${ }^{1} \mathrm{H}$ NMR ( $\left.500 \mathrm{MHz}, \mathrm{CDCl}_{3}\right) \delta: 1.25\left(\mathrm{t}, J 7.1 \mathrm{~Hz}, 3 \mathrm{H},-\mathrm{CH}_{3}\right), 1.35\left(\mathrm{~s}, 9 \mathrm{H},-\left(\mathrm{CH}_{3}\right)_{3}\right), 1.54(\mathrm{~d}, J 4.8 \mathrm{~Hz}, 3 \mathrm{H},-$ $\left.\mathrm{CH}_{3}\right)$, 4.06-4.09 (m, 2H, $\left.-\mathrm{CH}_{2}-\right), 5.71(\mathrm{q}, J 4.9 \mathrm{~Hz}, 1 \mathrm{H},-\mathrm{CH}-), 7.44(\mathrm{~d}, J 8.4 \mathrm{~Hz}, 2 \mathrm{H}, \mathrm{Ar}-\mathrm{H}), 7.67-7.71(\mathrm{~m}, 3 \mathrm{H}, \mathrm{Ar}-\mathrm{H})$, 


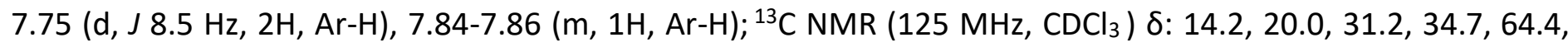
96.9, 101.3, 118.1, 124.24 (q, J 274 Hz, CF ), 125.5, 126.1, 127.8, 128.3, 129.2, 131.3, 132.3, 132.8, 151.8, 152.56, 160.5; HRMS (ESI) $m / z$ : Calculated, 462.1887, Found, $462.1892[\mathrm{M}+\mathrm{H}]^{+}$.

((2-(4-tert-Butylphenyl)-2-cyano-1-(2-trifluoromethylphenyl)vinyl)oxy)methyl cyclopropanecarboxylate 2 b. Yellow oil, yield 38\%, ${ }^{1} \mathrm{H}$ NMR ( $500 \mathrm{MHz}_{\mathrm{CDCl}}$ ) $\delta: 0.90-0.91$ (m, 2H, cyclopropyl), 0.95-0.96 (m, 2H, cyclopropyl), 1.34 (s, 9H, - $\left.\left(\mathrm{CH}_{3}\right)_{3}\right), 1.55-1.58(\mathrm{~m}, 1 \mathrm{H}, \mathrm{cyclopropyl}), 5.14+5.57$ (br, $\left.2 \mathrm{H},-\mathrm{CH}_{2}-\right), 7.44(\mathrm{~d}, J 8.4 \mathrm{~Hz}, 2 \mathrm{H}$, $\operatorname{Ar}-\mathrm{H})$, 7.62-7.73 (m, 1H, Ar-H), 7.68-7.76 (m, 4H, Ar-H), 7.84-7.86 (m, 1H, Ar-H); ${ }^{13} \mathrm{C} \mathrm{NMR}\left(125 \mathrm{MHz}^{\mathrm{C}} \mathrm{CDCl}_{3}\right) \delta:$ 9.2, 12.6, 31.2, 34.7, 84.6, 100.9, 118.1, 124.3 (q, J 274 Hz, CF $), 125.5,127.1,127.8,128.2,129.4,129.5,129.8$, 130.6, 131.4, 131.8, 131.8, 132.4, 132.8, 151.8, 161.0, 173.3; HRMS (ESI) $\mathrm{m} / \mathrm{z}$ : Calculated, 444.1781, Found, $444.1786[\mathrm{M}+\mathrm{H}]^{+}$.

1-((2-(4-tert-Butylphenyl)-2-cyano-1-(2-trifluoromethylphenyl)vinyl)oxy)ethyl cyclopropanecarboxylate 2c. Yellow oil, yield 42\%, ${ }^{1} \mathrm{H}$ NMR ( $\left.500 \mathrm{MHz}, \mathrm{CDCl}_{3}\right)$ 8: 0.85-0.92 (m, 4H, cyclopropyl), $1.35\left(\mathrm{~s}, 9 \mathrm{H},-\left(\mathrm{CH}_{3}\right)_{3}\right), 1.49(\mathrm{~d}$, J $\left.4.8 \mathrm{~Hz}, 3 \mathrm{H},-\mathrm{CH}_{3}\right), 1.59-1.62(\mathrm{~m}, 1 \mathrm{H}, \mathrm{cyclopropyl}), 5.84$ (q, J $\left.4.3 \mathrm{~Hz}, 1 \mathrm{H},-\mathrm{CH}-\right), 7.46(\mathrm{~d}, J 8.5 \mathrm{~Hz}, 2 \mathrm{H}, \mathrm{Ar}-\mathrm{H}), 7.64-$ $7.68(\mathrm{~m}, 3 \mathrm{H}, \mathrm{Ar}-\mathrm{H}), 7.78$ (d, J $8.5 \mathrm{~Hz}, 2 \mathrm{H}, \mathrm{Ar}-\mathrm{H}), 7.82(\mathrm{~d}, J 7.5 \mathrm{~Hz}, 1 \mathrm{H}, \mathrm{Ar}-\mathrm{H}) ;{ }^{13} \mathrm{C} \mathrm{NMR}\left(125 \mathrm{MHz}, \mathrm{CDCl}_{3}\right) \delta: 9.0$, 12.6, 20.0, 31.2, 34.7, 94.3, 101.0, 118.2, 124.3 (q, J 274 Hz, CF $), 125.5,127.1,128.0,128.3,129.7,130.5$, 131.3, 131.5, 132.3, 132.7, 151.7, 161.0, 172.7; HRMS (ESI) m/z: Calculated, 458.1938, Found, 458.1943 $[\mathrm{M}+\mathrm{H}]^{+}$.

1-((2-(4-tert-Butylphenyl)-2-cyano-1-(2-trifluoromethylphenyl)vinyl)oxy)ethyl pivalate 2d. Yellow oil, yield 48\%, ${ }^{1} \mathrm{H}$ NMR ( $\left.500 \mathrm{MHz}, \mathrm{CDCl}_{3}\right) \delta: 1.11\left(\mathrm{~s}, 9 \mathrm{H},-\left(\mathrm{CH}_{3}\right)_{3}\right), 1.35\left(\mathrm{~s}, 9 \mathrm{H},-\left(\mathrm{CH}_{3}\right)_{3}\right), 1.46\left(\mathrm{~d}, J 4.8 \mathrm{~Hz}, 3 \mathrm{H},-\mathrm{CH}_{3}\right), 5.83-$ 5.85 (br, 1H, -CH-), 7.44 (d, J 8.5 Hz, 2H, Ar-H), 7.66-7.69 (m, 3H, Ar-H), 7.77 (d, J 8.5 Hz, 2H, Ar-H), $7.83(\mathrm{~d}, J 7.2$ $\mathrm{Hz}, 1 \mathrm{H}, \mathrm{Ar}-\mathrm{H}) ;{ }^{13} \mathrm{C}$ NMR $\left(125 \mathrm{MHz}, \mathrm{CDCl}_{3}\right) \delta: 19.8,26.9,31.2,34.7,38.7,89.7,94.5,118.2,124.3$ (q, J $274 \mathrm{~Hz}$, $\left.\mathrm{CF}_{3}\right), 125.4,127.2,128.0,128.3,130.5,131.3,131.5,132.3,132.8,151.8,161.2,176.4$; HRMS (ESI) $\mathrm{m} / \mathrm{z}$ : Calculated, 474.2251, Found, $474.2256[\mathrm{M}+\mathrm{H}]^{+}$.

1-((2-(4-tert-Butylphenyl)-2-cyano-1-(2-trifluoromethylphenyl)vinyl)oxy)ethyl 2,2-dimethylbutanoate $2 \mathrm{e}$. Yellow oil, yield 45\%, ${ }^{1} \mathrm{H}$ NMR ( $500 \mathrm{MHz}, \mathrm{CDCl}_{3}$ ) $\delta: 0.75\left(\mathrm{t}, J 7.4 \mathrm{~Hz}, 3 \mathrm{H},-\mathrm{CH}_{3}\right), 1.06\left(\mathrm{~s}, 3 \mathrm{H},-\mathrm{CH}_{3}\right), 1.08(\mathrm{~s}, 3 \mathrm{H},-$ $\left.\mathrm{CH}_{3}\right), 1.35\left(\mathrm{~s}, 9 \mathrm{H},-\left(\mathrm{CH}_{3}\right)_{3}\right), 1.46\left(\mathrm{~d}, \mathrm{~J} 4.8 \mathrm{~Hz}, 3 \mathrm{H},-\mathrm{CH}_{3}\right), 1.58-1.61\left(\mathrm{~m}, 2 \mathrm{H},-\mathrm{CH}_{2}-\right), 5.86(\mathrm{br}, 1 \mathrm{H},-\mathrm{CH}-), 7.44(\mathrm{~d}, J 8.4$ $\mathrm{Hz}, 2 \mathrm{H}, \mathrm{Ar}-\mathrm{H}), 7.61-7.62(\mathrm{~m}, 1 \mathrm{H}, \mathrm{Ar}-\mathrm{H}), 7.66-7.68(\mathrm{~m}, 2 \mathrm{H}, \mathrm{Ar}-\mathrm{H}), 7.77(\mathrm{~d}, J 8.5 \mathrm{~Hz}, 2 \mathrm{H}, \mathrm{Ar}-\mathrm{H}), 7.83-7.84(\mathrm{~m}, 1 \mathrm{H}$, $\mathrm{Ar}-\mathrm{H}) ;{ }^{13} \mathrm{CNMR}\left(125 \mathrm{MHz}, \mathrm{CDCl}_{3}\right)$ ) $: 9.1,19.9,24.6,31.2,32.9,34.7,42.6,94.5,101.4,118.3,124.3$ (q, J $274 \mathrm{~Hz}$, $\left.\mathrm{CF}_{3}\right), 125.4,127.2,128.0,128.3,129.8,131.3,132.3,132.9,151.8,161.1,175.9 ; \mathrm{HRMS}$ (ESI) $\mathrm{m} / \mathrm{z}$ : Calculated, 488.2407, Found, $488.2410[\mathrm{M}+\mathrm{H}]^{+}$.

\section{1-((2-(4-tert-Butylphenyl)-2-cyano-1-(2-trifluoromethylphenyl)vinyl)oxy)ethyl (2,2,2-trifluoroethyl)}

\section{carbonate $2 f$.}

Yellow oil, yield 32\%, ${ }^{1} \mathrm{H}$ NMR ( $500 \mathrm{MHz}, \mathrm{CDCl}_{3}$ ) $\delta: 1.35\left(\mathrm{~s}, 9 \mathrm{H},-\left(\mathrm{CH}_{3}\right)_{3}\right), 1.54\left(\mathrm{~d}, \mathrm{~J} 4.8 \mathrm{~Hz}, 3 \mathrm{H},-\mathrm{CH}_{3}\right), 3.39(\mathrm{~s}, 3 \mathrm{H}$, $\left.-\mathrm{CH}_{3}\right), 3.56\left(\mathrm{t}, J 4.5 \mathrm{~Hz}, 2 \mathrm{H},-\mathrm{CH}_{2}-\right), 4.15\left(\mathrm{t}, J 4.5 \mathrm{~Hz}, 2 \mathrm{H},-\mathrm{CH}_{2}-\right), 5.71(\mathrm{q}, J 4.9 \mathrm{~Hz}, 1 \mathrm{H},-\mathrm{CH}-), 7.44(\mathrm{~d}, J 8.5 \mathrm{~Hz}, 2 \mathrm{H}$, $\mathrm{Ar}-\mathrm{H}), 7.67-7.71(\mathrm{~m}, 3 \mathrm{H}, \mathrm{Ar}-\mathrm{H}), 7.75(\mathrm{~d}, J 8.5 \mathrm{~Hz}, 2 \mathrm{H}, \mathrm{Ar}-\mathrm{H}), 7.84(\mathrm{~d}, J 7.4 \mathrm{~Hz}, 1 \mathrm{H}, \mathrm{Ar}-\mathrm{H}) ;{ }^{13} \mathrm{C} \mathrm{NMR}(125 \mathrm{MHz}$, $\mathrm{CDCl}_{3}$ ) $\delta: 19.9,31.2,34.7,59.0,67.2,69.9,97.1,101.3,118.1,122.4$ (q, J $\left.274 \mathrm{~Hz}, \mathrm{CF}_{3}\right), 124.26$ (q, J $\left.278 \mathrm{~Hz}, \mathrm{CF}_{3}\right)$, 125.5, 127.1, 127.7, 128.3, 129.5, 131.3, 132.4, 132.9, 151.8, 152.6, 160.4; HRMS (ESI) $\mathrm{m} / \mathrm{z}$ : Calculated, 516.1604, Found, $516.1609[\mathrm{M}+\mathrm{H}]^{+}$.

1-((2-(4-tert-Butylphenyl)-2-cyano-1-(2-trifluoromethylphenyl)vinyl)oxy)ethyl propyl carbonate 2g. Yellow oil, yield 33\%, ${ }^{1} \mathrm{H} N M R\left(500 \mathrm{MHz}, \mathrm{CDCl}_{3}\right.$ ) $\delta: 0.93\left(\mathrm{t}, \mathrm{J} 7.4 \mathrm{~Hz}, 3 \mathrm{H},-\mathrm{CH}_{3}\right), 1.35\left(\mathrm{~s}, 9 \mathrm{H},-\left(\mathrm{CH}_{3}\right)_{3}\right), 1.54(\mathrm{~d}, J 4.9 \mathrm{~Hz}, 3 \mathrm{H}$, $\left.-\mathrm{CH}_{3}\right), 1.62-1.65\left(\mathrm{~m}, 2 \mathrm{H},-\mathrm{CH}_{2}-\right)$, 3.95-3.99 (m, 2H, $\left.-\mathrm{CH}_{2-}\right), 5.71(\mathrm{q}, J 4.9 \mathrm{~Hz}, 1 \mathrm{H},-\mathrm{CH}-), 7.44(\mathrm{~d}, J 8.5 \mathrm{~Hz}, 2 \mathrm{H}, \mathrm{Ar}-\mathrm{H})$, 7.67-7.70 (m, 3H, Ar-H), 7.75 (d, J $8.5 \mathrm{~Hz}, 2 \mathrm{H}, \mathrm{Ar}-\mathrm{H}), 7.84-7.85(\mathrm{~m}, 1 \mathrm{H}, \mathrm{Ar}-\mathrm{H}) ;{ }^{13} \mathrm{C} \mathrm{NMR}\left(125 \mathrm{MHz}, \mathrm{CDCl}_{3}\right) \delta: 10.1$, 20.0, 21.9, 31.2, 34.7, 70.0, 96.9, 101.3, 118.1, 124.3 (q, J 274 Hz, CF $), 125.5,127.1,127.8,128.3,129.6,131.3$, 132.3, 132.8, 151.8, 152.7, 160.5; HRMS (ESI) m/z: Calculated, 476.2043, Found, $476.2048[\mathrm{M}+\mathrm{H}]^{+}$. 
1-((2-(4-tert-Butylphenyl)-2-cyano-1-(2-trifluoromethylphenyl)vinyl)oxy)ethyl isobutyl carbonate $2 \mathrm{~h}$. Yellow oil, yield 46\%, ${ }^{1} \mathrm{H}$ NMR $\left(500 \mathrm{MHz}, \mathrm{CDCl}_{3}\right) \delta: 0.92\left(\mathrm{~d}, J 1.96 \mathrm{~Hz}, 3 \mathrm{H},-\mathrm{CH}_{3}\right), 0.94\left(\mathrm{~d}, J 1.9 \mathrm{~Hz}, 3 \mathrm{H},-\mathrm{CH}_{3}\right), 1.35(\mathrm{~s}, 9 \mathrm{H}$, $\left.-\left(\mathrm{CH}_{3}\right)_{3}\right), 1.54\left(\mathrm{~d}, J 5.0 \mathrm{~Hz}, 3 \mathrm{H},-\mathrm{CH}_{3}\right), 1.89-1.93(\mathrm{~m}, 1 \mathrm{H},-\mathrm{CH}-), 3.76-3.81\left(\mathrm{~m}, 2 \mathrm{H},-\mathrm{CH}_{2}-\right), 5.71(\mathrm{q}, J 4.9 \mathrm{~Hz}, 1 \mathrm{H},-\mathrm{CH}-)$, $7.44(\mathrm{~d}, J 8.4 \mathrm{~Hz}, 2 \mathrm{H}, \mathrm{Ar}-\mathrm{H}), 7.67-7.70(\mathrm{~m}, 3 \mathrm{H}, \mathrm{Ar}-\mathrm{H}), 7.76(\mathrm{~d}, J 8.4 \mathrm{~Hz}, 2 \mathrm{H}, \mathrm{Ar}-\mathrm{H}), 7.84-7.85(\mathrm{~m}, 1 \mathrm{H}, \mathrm{Ar}-\mathrm{H}) ;{ }^{13} \mathrm{C}$

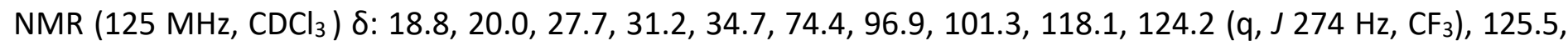
127.1, 127.8, 128.3, 129.6, 131.3, 132.3, 132.8, 151.8, 152.8, 160.5; HRMS (ESI) $\mathrm{m} / \mathrm{z}$ : Calculated, 490.2200, Found, $490.2200[\mathrm{M}+\mathrm{H}]^{+}$.

Butyl (1-((2-(4-tert-butylphenyl)-2-cyano-1-(2-trifluoromethylphenyl)vinyl)oxy)ethyl) carbonate 2i. Yellow oil, yield 47\%, ${ }^{1} \mathrm{H}$ NMR ( $\left.500 \mathrm{MHz}, \mathrm{CDCl}_{3}\right) \delta: 0.92\left(\mathrm{t}, \mathrm{J} 7.4 \mathrm{~Hz}, 3 \mathrm{H},-\mathrm{CH}_{3}\right), 1.35\left(\mathrm{~s}, 9 \mathrm{H},-\left(\mathrm{CH}_{3}\right)_{3}\right), 1.36-1.39\left(\mathrm{~m}, 2 \mathrm{H},-\mathrm{CH}_{2}-\right.$ ), $1.54\left(\mathrm{~d}, J 5.0 \mathrm{~Hz}, 3 \mathrm{H},-\mathrm{CH}_{3}\right), 1.58-1.61\left(\mathrm{~m}, 2 \mathrm{H},-\mathrm{CH}_{2}-\right), 4.00\left(\mathrm{q}, J 6.4 \mathrm{~Hz}, 2 \mathrm{H},-\mathrm{CH}_{2}-\right), 5.71(\mathrm{q}, J 4.9 \mathrm{~Hz}, 1 \mathrm{H},-\mathrm{CH}-)$, $7.44(\mathrm{~d}, J 8.5 \mathrm{~Hz}, 2 \mathrm{H}, \mathrm{Ar}-\mathrm{H}), 7.67-7.70(\mathrm{~m}, 3 \mathrm{H}, \mathrm{Ar}-\mathrm{H}), 7.76(\mathrm{~d}, J 8.5 \mathrm{~Hz}, 2 \mathrm{H}, \mathrm{Ar}-\mathrm{H}), 7.84-7.85(\mathrm{~m}, 1 \mathrm{H}, \mathrm{Ar}-\mathrm{H}) ;{ }^{13} \mathrm{C}$ NMR $\left(125 \mathrm{MHz}_{1} \mathrm{CDCl}_{3}\right)$ ): 13.6, 18.8, 20.0, 30.5, 31.2, 34.7, 68.3, 96.9, 101.3, 118.1, 124.3 (q, J 274 Hz, CF3), 125.5, 127.1, 127.8, 128.3, 129.6, 131.3, 132.3, 132.9, 151.8, 152.7, 160.5; HRMS (ESI) $\mathrm{m} / \mathrm{z}$ : Calculated, 490.2200, Found, $490.2205[\mathrm{M}+\mathrm{H}]^{+}$.

1-((2-(4-tert-Butylphenyl)-2-cyano-1-(2-trifluoromethylphenyl)vinyl)oxy)ethyl methyl carbonate 2j. Yellow oil, yield 44\%, ${ }^{1} \mathrm{H}$ NMR ( $500 \mathrm{MHz}, \mathrm{CDCl}_{3}$ ) $\delta: 1.35\left(\mathrm{~s}, 9 \mathrm{H},-\left(\mathrm{CH}_{3}\right)_{3}\right), 1.54\left(\mathrm{~d}, J 4.8 \mathrm{~Hz}, 3 \mathrm{H},-\mathrm{CH}_{3}\right), 3.68\left(\mathrm{~s}, 3 \mathrm{H},-\mathrm{CH}_{3}\right)$, $5.73(\mathrm{~s}, 1 \mathrm{H},-\mathrm{CH}-), 7.45$ (d, J $8.5 \mathrm{~Hz}, 2 \mathrm{H}, \mathrm{Ar}-\mathrm{H}), 7.67-7.70(\mathrm{~m}, 3 \mathrm{H}, \mathrm{Ar}-\mathrm{H}), 7.75(\mathrm{~d}, J 8.5 \mathrm{~Hz}, 2 \mathrm{H}, \mathrm{Ar}-\mathrm{H}), 7.84-7.86(\mathrm{~m}$,

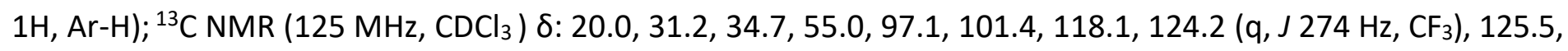
127.1, 127.7, 128.3, 129.5, 131.4, 132.3, 132.8, 151.8, 153.2, 160.4; HRMS (ESI) m/z: Calculated, 448.1730, Found, 448.1735[M+H] $]^{+}$.

Allyl (1-((2-(4-tert-butylphenyl)-2-cyano-1-(2-trifluoromethylphenyl)vinyl)oxy)ethyl) carbonate 2k. Yellow oil, yield 41\%, ${ }^{1} \mathrm{H}$ NMR ( $\left.500 \mathrm{MHz}, \mathrm{CDCl}_{3}\right) \delta: 1.35\left(\mathrm{~s}, 9 \mathrm{H},-\left(\mathrm{CH}_{3}\right)_{3}\right), 1.54\left(\mathrm{~d}, \mathrm{~J} 5.1 \mathrm{~Hz}, 3 \mathrm{H},-\mathrm{CH}_{3}\right), 4.47-4.51(\mathrm{~m}, 2 \mathrm{H},-$ $\left.\mathrm{CH}_{2}-\right)$, 5.28-5.35 (m, 2H, $\left.-\mathrm{CH}=\mathrm{CH}_{2}\right), 5.72(\mathrm{q}, J 4.9 \mathrm{~Hz}, 1 \mathrm{H},-\mathrm{CH}-), 5.83-5.87\left(\mathrm{~m}, 1 \mathrm{H},-\mathrm{CH}=\mathrm{CH}_{2}\right), 7.45(\mathrm{~d}, J 8.5 \mathrm{~Hz}, 2 \mathrm{H}$, $\mathrm{Ar}-\mathrm{H})$, 7.66-7.68 (m, 3H, Ar-H), $7.75(\mathrm{~d}, J 8.5 \mathrm{~Hz}, 2 \mathrm{H}, \mathrm{Ar}-\mathrm{H}), 7.83-7.85(\mathrm{~m}, 1 \mathrm{H}, \mathrm{Ar}-\mathrm{H}) ;{ }^{13} \mathrm{C} \mathrm{NMR}\left(125 \mathrm{MHz}, \mathrm{CDCl}_{3}\right) \delta$ : 19.9, 31.2, 34.7, 68.7, 97.0, 101.4, 118.1, 119.5, 124.3 (q, J 274 Hz, CF $), 125.5,127.1,127.7,128.3,130.9$, 131.3, 132.4, 132.8, 151.8, 152.4, 160.4; HRMS (ESI) m/z: Calculated, 474.1887, Found, $474.1892[\mathrm{M}+\mathrm{H}]^{+}$.

\section{Acaricidal activity}

All bioassays were performed on representative test organisms reared in the laboratory or greenhouse. The bioassay was repeated at $25 \pm 1^{\circ} \mathrm{C}$ according to statistical requirements.

Lab test. The acaricidal activities of the title compounds $\mathbf{2 a - 2} \mathbf{g}$ were tested against Spider Mites (Tetranychus cinnabarinus). The leaves of horse bean plants were punched into $3 \mathrm{~cm}$-diameter discs, using leaf punch, and placed onto moist filter paper in a $7 \mathrm{~cm}$-diameter styrol cup. Ten larvae spider mites (Tetranychus cinnabarinus)were put onto each leaf. A $5 \%$ emulsion of a compound was diluted with water containing a spreading agent to give a 1-10 ppm solution of the compound. The solution was sprayed over each cup in an amount of $2 \mathrm{~mL} / \mathrm{cup}$, using a rotary sprinkler. After $48 \mathrm{~h}$, the mites in each cup were observed, and mortality of the mites was determined. Assessments were made on a dead/alive basis, and mortality rates were corrected using Abbott's formula. Evaluations are based on a percentage scale of 0-100 in which $0=$ no activity and $100=$ total kill. The results of acaricidal activity are listed in Table 1.

Greenhouse test. The greenhouse test against Tetranychus cinnabarinus was carried out in horse bean plants. One bean seedlings with 2 to 3 broad leaves were transplanted into a plastic bowl with a diameter of $9 \mathrm{~cm}$, and the seedlings were inoculated with Tetranychus cinnabarinus. Each pot of seedlings is a plot, and each concentration was repeated 3 times, randomly arranged, and clear water was used as a blank control. The number of spider mites on each plant was investigated, recorded and calculated to give a relative efficacy (\%) 
after 4 days, 7 days and 14 days. The spray method was used to spray broad bean seedlings with a spray volume of $2 \mathrm{~mL}$ per treatment.

\section{Molecular Docking}

The molecular docking simulations were carried to analyze interactions using Discovery Studio 2.5 software according to the reported method. ${ }^{42,43}$ From the mechanism of cyflumetofen, the cyflumetofen is metabolised in vivo to the acarcidal active intermediate 3-oxo-2-phenyl-3-(2-trifluoromethylphenyl)propanenitrile. ${ }^{40}$ Accoridngly, the structure of intermediate 2-(4-tert-butylphenyl)-3-oxo-3-(2trifluoromethylphenyl)propanenitrile 1 was selected as ligand and input into the Discovery Studio 2.5 software. The procine SDH structure (PDB code: 1ZOY) was selected for docking simulation and binding sites were generated from the protein complex. CHARMM and MMFF94 force fields were applied in compound and SDH. Then the docking process was carried out by using CDOCKER protocol. Finally, docking scores (-CDOCKER interaction energy) of the best-fitted interactions with the active site at the SDH structure were recorded.

\section{Acknowledgements}

This work was funded by Zhejiang Provincial Natural Science Foundation of China (No. LY19C140002) and Research Fund of Department of Education of Zhejiang Province (Y201941832).

\section{Supplementary Material}

${ }^{1} \mathrm{H}$ NMR spectra for compound 1 and full ${ }^{1} \mathrm{H}$ NMR, ${ }^{13} \mathrm{C}$ NMR and HRMS spectra for compounds $2 \mathrm{a}-\mathbf{k}$ can be found via the "Supplementary Material" section of this article's online webpage.

\section{References}

1. Dai, H.; Ge, S. S.; Guo, J.; Chen, S.; Huang, M. L.; Yang, J. Y.; Sun, S. Y.; Ling, Y.; Shi, Y. J., Eur. J. Med. Chem. 2018, 143, 1066-1076. https://doi.org/10.1016/i.ejmech.2017.11.098

2. Padalkar, V. S.; Borse, B. N.; Gupta, V. D.; Phatangare, K. R.; Patil, V. S.; Sekar, N. J. Heterocycl. Chem. 2016, 53, 1347-1355.

https://doi.org/10.1002/ihet.1506

3. Radi, S.; Attayibat, A.; El-Massaoudi, M.; Salhi, A.; Eddike, D.; Tillard, M.; Mabkhot, Y.N. Molecules 2016, 21, 1020.

https://doi.org/10.3390/molecules21081020

4. Cheng, L.; Zhao, W.; Shen, Z. H.; Xu, T. M.; Wu, H. K.; Peng, W. L.; Liu, X. H. Lett. Drug Des. Discov. 2019, 16, 29-35. https://doi.org/10.2174/1570180815666180326150827

5. Zhao, W.; Xing, J. H.; Xu, T. M.; Peng, W. L.; Liu, X. H. Front. Chem. Sci. Eng. 2017, 11, 363-368. https://doi.org/10.1007/s11705-016-1595-x 
6. Zhao, W.; Shen, Z.H.; Xu, T. M.; Peng, W. L.; Liu, X. H. J. Heterocycl. Chem. 2017, 54, 1751-1756. https://doi.org/10.1002/jhet.2753

7. Zhao, W.; Shen, Z. H.; Xing, J. H.; Yang, G.; Xu, T. M.; Peng, W. L.; Liu, X. H. Chem. Pap. 2017, 71, $921-928$. https://doi.org/10.1007/s11696-016-0012-8

8. Zhao, W.; Shen, Z. H.; Xing, J. H.; Xu, T. M.; Peng, W. L.; Liu, X. H. Chin. J. Struct. Chem. 2017, 36, 423-428. https://doi.org/10.14102/j.cnki.0254-5861.2011-1353

9. Cheng, L.; Cai, P. P.; Zhang, R. R.; Han, L.; Tan, C. X.; Weng, J. Q.; Xu, T. M.; Liu, X. H. J. Heterocycl. Chem. 2019, 56, 1312-1317. https://doi.org/10.1002/ihet.3502

10. Liu, X. H.; Zhao, W.; Shen, Z. H.; Xing, J. H.; Yuan, J.; Yang, G.; Xu, T. M.; Peng, W. L. Bioorg. Med. Chem. Lett. 2016, 26, 3626-3628. https://doi.org/10.1016/i.bmcl.2016.06.004

11. Sun, N. B.; Shen, Z. H.; Zhai, Z. W.; Han, L.; Weng, J. Q.; Tan, C. X.; Liu, X. H. Chin. J. Org. Chem. 2017, 37, 2705-2710. https://doi.org/10.6023/cjoc201702003

12. Liu, X. H,; Qiao, L.; Zhai, Z. W.; Cai, P. P.; Cantrell, C. L.; Tan, C. X.; Weng, J. Q.; Han, L.; Wu, H. K. Pest Manag. Sci. 2019, 75, 2892-2900. https://doi.org/10.1002/ps.5463

13. Zhao, W.; Shen, Z. H.; Xing, J. H.; Xu, T. M.; Peng, W. L.; Liu, X. H. Lett. Drug Des. Discov. 2017, 14, $323-329$. https://doi.org/10.2174/1570180813666160930164327

14. Jin, T.; Zhai, Z. W.; Han, L.; Weng, J. Q.;Tan, C. X.; Liu, X. H. Chin. J. Struct. Chem. 2018, 37, 1259-1264. https://doi.org/10.14102/j.cnki.0254-5861.2011-1919

15. Liu, X. H.; Tan, C. X.; Weng, J. Q. Phosphorus Sulfur Silicon Relat. Elem. 2011, 186, 552-557. https://doi.org/10.1080/10426507.2010.508059f

16. Liu, X. H.; Zhao, W.; Shen, Z. H.; Xing, J. H.; Xu, T. M.; Peng, W. L. Eur. J. Med. Chem. 2017, 125, 881-889. https://doi.org/10.1016/i.ejmech.2016.10.017

17. Sun, N. B.; Zhai, Z. W.; Tong, J. Y.; Cai, P. P.; He, F. Y.; Han, L.; Liu, X. H. Chin. J. Struct. Chem. 2019, 38, 706712.

https://doi.org/10.14102/i.cnki.0254-5861.2011-2176

18. Wang, H.; Zhai, Z. W.; Shi, Y. X.; Tan, C. X.; Weng, J. Q.; Han, L.; Li, B. J.; Liu, X. H. Lett. Drug Des. Discov. 2019, 16, 785-791.

https://doi.org/10.2174/1570180815666180704103047

19. Wang, H.; Zhai, Z. W.; Shi, Y. X.; Tan, C. X.; Weng, J. Q.; Han, L.; Li, B. J.; Liu, X. H. J. Mol. Struct. 2018, 1171, 631-638.

https://doi.org/10.1016/i.molstruc.2018.06.050

20. Cheng, L.; Zhang, R. R.; Wu, H. K.; Xu, T. M.; Liu, X. H. Front. Chem. Sci. Eng. 2019, 13, 369-376. https://doi.org/10.1007/s11705-018-1734-7

21. Hayashi, N.; Sasama, Y.; Takahashi, N.; Ikemi, N. Pest Manag. Sci. 2013, 69, 1080-1084. https://doi.org/10.1002/ps.3470

22. Liu, X. H.; Wang, Q.; Sun, Z. H.; Wedge, D. E.; Becnel, J. J.; Estep, A. S.; Tan, C. X.; Weng, J. Q. Pest Manag. Sci. 2017, 73, 953-959. https://doi.org/10.1002/ps.4370

23. Liu, X. H.; Weng, J. Q.; Wang, B. L.; Li, Y. H.; Tan, C. X.; Li, Z. M. Res. Chem. Intermed. 2014, 40, $2605-2612$. https://doi.org/10.1007/s11164-012-0521-1 
24. Shen, Z. H.; Sun, Z. H.; Becnel, J. J.; Estep, A.; Wedge, D. E.; Tan, C. X.; Weng, J. Q.; Han, L, Liu. X. H. Lett. Drug Des. Discov. 2018, 15, 951-956. https://doi.org/10.2174/1570180815666180102141640

25. Liu, X. H.; Pan, L.; Weng, J. Q.; Tan, C. X.; Li, Y. H.; Wang, B. L.; Li, Z. M. Mol. Divers. 2012, 16, 251-260. https://doi.org/10.1007/s11030-011-9352-z

26. Tan, C. X.; Shi, Y. X.; Weng, J. Q.; Liu, X. H.; Li, B. J.; Zhao, W. G. Lett. Drug Des. Discov. 2012, 9, 431-435. https://doi.org/10.2174/157018012799859954

27. Zhang, L. J.; Yang, M. Y.; Sun, Z. H.; Tan, C. X.; Weng, J. Q.; Wu, H. K.; Liu, X. H. Lett. Drug Des. Discov. 2014, 11, 1107-1111. https://doi.org/10.2174/1570180811666140610212731

28. Weng, J. Q.; Wang, L.; Liu, X. H. J. Chem. Soc. Pak. 2012, 34, 1248-1252.

29. Liu, X. H.; Zhai, Z. W.; Xu, X. Y.; Yang, M. Y.; Sun, Z. H.; Weng, J. Q.; Tan, C. X.; Chen, J. Bioorg. Med. Chem. Lett. 2015, 25, 5524-5528.

https://doi.org/10.1016/j.bmcl.2015.10.064

30. Liu, X. H.; Xu, X. Y.; Tan, C. X.; Weng, J. Q.; Xin, J. H.; Chen, J. Pest Manag. Sci. 2015, 71, 292-301. https://doi.org/10.1002/ps.3804

31. Zhang, L. J.; Yang, M. Y.; Hu, B. Z.; Sun, Z. H.; Liu, X. H.; Weng, J. Q.; Tan, C. X. Turk. J. Chem. 2015, 39, 867873.

https://doi.org/10.3906/kim-1408-78

32. Yang, M. Y.; Zhao, W.; Sun, Z. H.; Tan, C. X.; Weng, J. Q.; Liu, X. H. Lett. Drug Des. Discov. 2015, 12, 314318.

https://doi.org/10.2174/1570180811666141010000435

33. Liu, X. H.; Weng, J. Q.; Wang, B. L.; Li, Y. H.; Tan, C. X.; Li, Z. M. Res. Chem. Intermed. 2014, 40, $2605-2612$. https://doi.org/10.1007/s11164-013-1113-4

34. Yan, S. L.; Yang, M. Y.; Sun, Z. H.; Min, L. J.; Tan, C. X.; Weng, J. Q.; Wu, H. K.; Liu, X. H. Lett. Drug Des. Discov. 2014, 11, 940-943. https://doi.org/10.2174/1570180811666140423222141

35. Liu, X. H.; Tan, C. X.; Weng, J. Q. Phosphorus Sulfur Silicon Relat. Elem. 2011, 186, 558-564. https://doi.org/10.1080/10426507.2010.508060

36. Min, L. J.; Zhai, Z. W.; Shi, Y. X.; Han, L.; Tan, C. X.; Weng, J. Q.; Li, B. J.; Zhang, Y. G.; Liu, X. H. Phosphorus Sulfur Silicon Relat. Elem. 2019, https://doi.org/10.1080/10426507.2019.1633530

37. Liu, X. H.; Fang, Y. M.; Xie, F.; Zhang, R. R.; Shen, Z. H.; Tan, C. X.; Weng, J. Q.; Xu, T. M.; Huang, H. Y. Pest Manag. Sci. 2017, 73, 1900-1907. https://doi.org/10.1002/ps.4556

38. Fang, Y. M.; Zhang, R. R.; Shen, Z. H.; Tan, C. X.; Weng, J. Q.; Xu, T. M.; Liu, X. H. Huang, H. Y.; Wu, H. K. Lett. Drug Des. Discov. 2018, 15, 1314-1318. https://doi.org/10.2174/1570180815666180313121735

39. Fang, Y. M.; Zhang, R. R.; Shen, Z. H.; Wu, H. K.; Tan, C. X.; Weng, J. Q.; Xu, T. M.; Liu, X. H. J. Heterocycl. Chem. 2018, 55, 240-245.

https://doi.org/10.1002/jhet.3031

40. Kaisha, I. S.; Toru, K.; Yuji, N.; Tsuyoshi, U.; Arkishiro, H.; Mayumi, K. WO0109086A1. 
41. Huang, D. L.; Huang, M. Z.; Liu, A. P.; Liu, X. P.; Liu, W. D.; Chen, X. Y.; Xue, H. S.; Sun, J.; Yin, D. L.; Wang, X. G. J. Heterocycl. Chem. 2017, 54, 1121-1128.

https://doi.org/10.1002/jhet.2682

42. Fu, Q.; Cai, P. P.; Cheng, L.; Zhong, L. K.; Tan, C. X.; Shen, Z. H.; Han, L.; Xu, T. M.; Liu, X. H. Pest Manag. Sci. 2019,

https://doi.org/10.1002/ps.5591

43. Fu, Y.; Zhang, S. Q.; Liu, Y. X.; Wang, J. Y.; Gao, S.; Zhao, L. X.; Ye, F. Ind. Crop Prod. 2019; 137, $566-575$. https://doi.org/10.1016/i.indcrop.2019.05.070

This paper is an open access article distributed under the terms of the Creative Commons Attribution (CC BY) license (http://creativecommons.org/licenses/by/4.0/ 